\title{
Population screening and cascade testing for carriers of SMA
}

\author{
Melanie Smith, Vanessa Calabro, Belinda Chong, Nicole Gardiner, Shannon Cowie and \\ Desirée du Sart*
}

Molecular Genetics Laboratory, Victorian Clinical Genetics Services, Murdoch Childrens Research Institute, Parkville, Victoria, Australia

Spinal muscular atrophy (SMA) is one of the most common autosomal-recessive diseases, caused by absence of both copies of the survival motor neuron 1 (SMN1) gene. Identification of SMA carriers has important implications for individuals with a family history and the general population. SMA carriers are completely healthy and most are unaware of their carrier status until they have an affected child. A total of 422 individuals have been studied to identify SMA carriers. This cohort included 117 parents of children homozygously deleted for SMN1 (94\% were carriers and 6\% had two copies of SMN1; of these individuals, two in seven had the ' $2+0$ ' genotype, two in seven were normal but had children carrying a de novo deletion and three in seven were unresolved), 158 individuals with a significant family history of SMA (47\% had one copy, 49\% had two copies and 4\% had three copies of SMN1) and 146 individuals with no family history of SMA (90\% had two copies, $2 \%$ had one copy and $8 \%$ had three copies of SMN1). The SMA carrier frequency in the Australian population appears to be 1/49 and the frequency of two-copy SMN1 alleles and de novo deletion mutations are both at least $1.7 \%$. A multimodal approach involving quantitative analysis, linkage analysis and genetic risk assessment (GRA), facilitates the resolution of SMA carrier status in individuals with a family history as well as individuals of the general population, providing couples with better choices in their family planning.

European Journal of Human Genetics (2007) 15, 759-766; doi:10.1038/sj.ejhg.5201821; published online 28 March 2007

Keywords: spinal muscular atrophy; carrier testing; SMN1; risk assessment; population screening; cascade testing

Introduction

Spinal muscular atrophy (SMA) is the second most common severe autosomal-recessive disorder after cystic fibrosis and the most frequent genetic cause of infant mortality. SMA has an estimated incidence of $1 / 10000$ live births with a carrier frequency of $1 / 40-1 / 50 .^{1}$ It is

*Correspondence: Dr D du Sart, Molecular Genetics Laboratory, Murdoch Childrens Research Institute, 10th Floor Royal Children's Hospital, Flemington Road, Parkville, Victoria, 3052, Australia.

Tel: + 613 83416333; Fax: + 61383416366 ;

E-mail: desiree.dusart@mcri.edu.au

Received 3 January 2007; revised 13 February 2007; accepted 21 February 2007; published online 28 March 2007 characterized by degeneration of the anterior horn cells of the spinal cord, leading to symmetrical muscle weakness and atrophy. The SMA determining gene was identified in $1995^{2}$ and is named the survival motor neuron 1 (SMN1) gene. The SMN1 gene is localized to chromosome region $5 q 13$ within a highly complex, volatile area containing multiple repetitive and inverted sequences.

There is a highly homologous gene copy to SMN1 called SMN2, which differs by only a single nucleotide in the coding sequence of exon $7 .^{3}$ This single base $\mathrm{C}$ to $\mathrm{T}$ substitution is responsible for exon 7 skipping in most SMN2 products, either due to the creation of an exonic splicing silencer ${ }^{4}$ or disruption of an exonic splicing 
enhancer, ${ }^{5}$ which results in the majority of SMN2 transcripts lacking exon 7. SMN2 is responsible for mediating the severity of the SMA clinical phenotype. ${ }^{6}$ Approximately, 94\% of clinically typical SMA patients lack both copies of SMN1. The homozygous absence of SMN1 is caused by deletions or gene conversions of sequences of SMN1 into SMN2.7,8 The remaining 6\% of affected individuals who do not lack both copies of SMN1 may be compound heterozygous patients who have a deletion on one allele and a point mutation on the other ${ }^{9}$ or patients with SMA phenotypes unrelated to SMN1. Currently at least 30 small intragenic mutations have been reported in SMN1. ${ }^{10}$

The copy number of SMN1 varies greatly in different individuals. The majority of affected individuals have no copies of SMN1, most carriers have one copy of SMN1 and non-carriers can have two to four copies of SMN1. In many instances, people do not know that they are carriers until they have a child affected with SMA. In addition, there are asymptomatic individuals who are homozygously deleted for SMN1 in some families, but have multiple copies of SMN2. It is believed that five or more copies of SMN2 could be enough to correct or partially compensate for the absence of the SMN1 gene. ${ }^{11}$

In this study, we describe the complexities in SMA carrier testing and discuss the implications for population screening of carriers of SMA. We have developed a method to determine the SMN1 copy number using quantitative real time polymerase chain reaction (PCR) that defines specific, non-overlapping ranges for 1,2 and 3 copy numbers of the SMN1 gene.

\section{Materials and methods Patient samples}

SMA carrier testing was performed on 117 parents of affected children lacking both copies of SMN1, 158 unaffected individuals with a family history of SMA and 147 unaffected individuals without a family history of SMA (population screen). Informed consent was obtained from all subjects and ethics approval was not required because testing was performed for clinical reasons. DNA was isolated from EDTA blood samples by the Nucleon BACC2 Kit (Amersham Biosciences UK Ltd, Little Chalfont, Buckinghamshire, England), or by external laboratories referring samples in for testing.

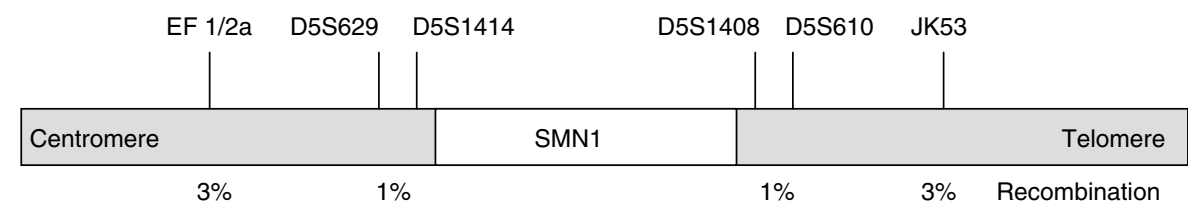

Figure 1 Genetic markers flanking the SMN1 gene.

\section{Quantitative real-time PCR}

Probes and specific primers that distinguish between SMN1 and SMN2 were designed using the program Primer Express (ABI, Foster City, CA, USA). A multiplex PCR simultaneously amplifying SMN1 and the CFTR gene (internal standard) was performed in a total volume of $25 \mu \mathrm{l}$, containing $200 \eta \mathrm{g}$ of DNA, $15 \mu \mathrm{M}$ of SMN primers (SMN1F TCCTTTTATTTTCCTTACAGGGTTTC and SMN1-R GCTGGCAGACTTACTCCTTAATTTAA), $5 \mu \mathrm{M}$ of CFTR primers (CFTR-F GAAGAGAACAAAGTGCGGCAG and CFTR-R TTGCCGGAAGAGGCTCCT), $12.5 \mu \mathrm{l}$ of Platinum Quantitative PCR Supermix-UDG (Invitrogen, Karlsruhe, Germany), $5 \mu \mathrm{M}$ of the SMN probe (ACAAAATCAAA AAGAAGGAAGGTGCTCACATTC) and $10 \mu \mathrm{M}$ of the CFTR probe (ACGATTCCATCCAGAAACTGCTGAACGA) on the BIORAD i-Cycler instrument. Each control and test sample was tested in triplicate within the same assay. Ten controls were included in each assay; these included a no DNA control, three one-copy SMN1 controls, five two-copy SMN1 controls and one no-copy SMN1 sample. The thermal cycling conditions included an initial denaturation of $10 \mathrm{~min}$ at $95^{\circ} \mathrm{C}$, and proceeded with 40 cycles of $15 \mathrm{~s}$ at $95^{\circ} \mathrm{C}$ and $1 \mathrm{~min}$ at $60^{\circ} \mathrm{C}$.

Data evaluation was carried out using i-Cycler iQ Real Time Detection System Software (Version 3) BIORAD. The formula $2^{-\Delta \Delta C \mathrm{t}}$ was used to determine the SMN1 gene copy number: where $\Delta \Delta C \mathrm{t}=(\mathrm{SMN} \Delta C \mathrm{t}-\mathrm{CFTR} \Delta C \mathrm{t})-$ Ave $\Delta C \mathrm{t}$ of the two-copy SMN1 controls. The $\Delta C \mathrm{t}$ represents the mean $C t$ (threshold cycle) value of each sample (average of triplicates). Values were standardized against the two-copy SMN1 control samples. Samples were repeated if the realtime values were situated between ranges or the standard deviation (SD) between the triplicates was $>0.3$.

\section{Linkage analysis}

Molecular analysis with polymorphic markers flanking the SMN gene was performed as described previously, using the distal markers JK53, D5S610 and D5S1408 and proximal markers EF1/2a, D5S629 and D5S1414. ${ }^{12}$ See Figure 1.

\section{Results}

\section{SMN1 gene dosage validation}

To validate the assay, we analyzed 53 carriers (parents of SMN1 homozygously deleted children) and 52 controls with quantitative real time PCR. In addition, a number of 


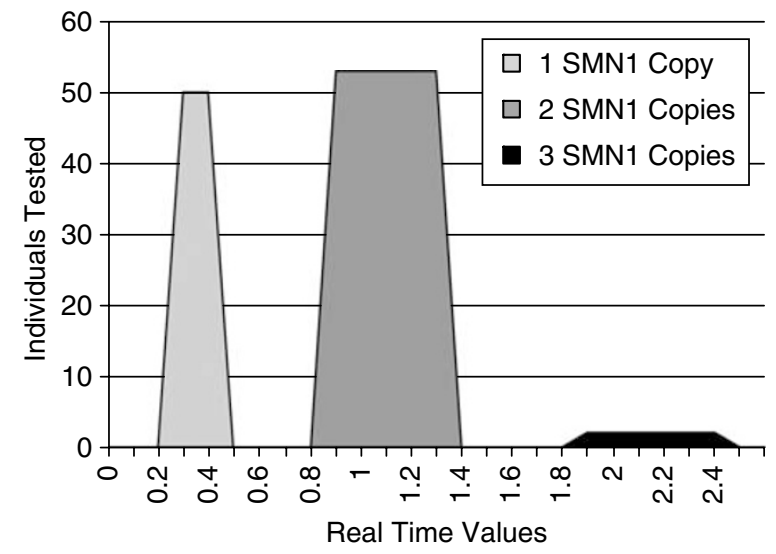

Figure 2 Validation of SMN1 gene dosage for SMA carrier testing.

samples used in the validation experiment were tested with a second independent quantitative method. Ten no-copy samples, 11 1-copy samples, 14 2-copy samples and six 3-copy samples for the SMN1 gene were screened with a Multiplex Ligation-dependent Probe Amplification assay, the SALSA MLPA kit P021 (MRC-Holland, The Netherlands). These samples were confirmed to have zero, one, two and three copies of exon 7 of the SMN1 gene (respectively) (data not shown). The quantitative real time PCR method defined specific, non-overlapping ranges that clearly distinguished between one, two or three SMN1 copies, as shown in Figure 2.

\section{SMN1 gene dosage analysis}

A total of 422 individuals from Australia and New Zealand have been studied to determine the SMN1 copy number as summarised in Figure 3. There were 147 unaffected individuals tested with no family history of SMA, which included a collection of random control samples and partners of individuals with a family history of SMA. In this group, three individuals had one copy of SMN1, 133 had two copies of SMN1 and 11 had three copies of SMN1. There were 158 individuals with a family history of suspected or definitive chromosome 5q13 linked SMA. Of these, 75 had one copy of SMN1, 77 had two copies of SMN1 and six had three copies of SMN1. Among the parents, 110 of 117 had one copy of SMN1, confirming their SMA carrier status as expected, and seven parents had two-copies of SMN1, which required further linkage analysis to determine the gene copy number on each allele.

\section{SMA linkage analysis}

In families 1 and 2, the fathers were found to have two copies of SMN1 (Figure 4). Combined dosage and linkage analyses indicated that the fathers have one SMN1 copy on each allele. Therefore, there was loss of the paternal SMN1 gene on transmission from the father to child, a de novo event.

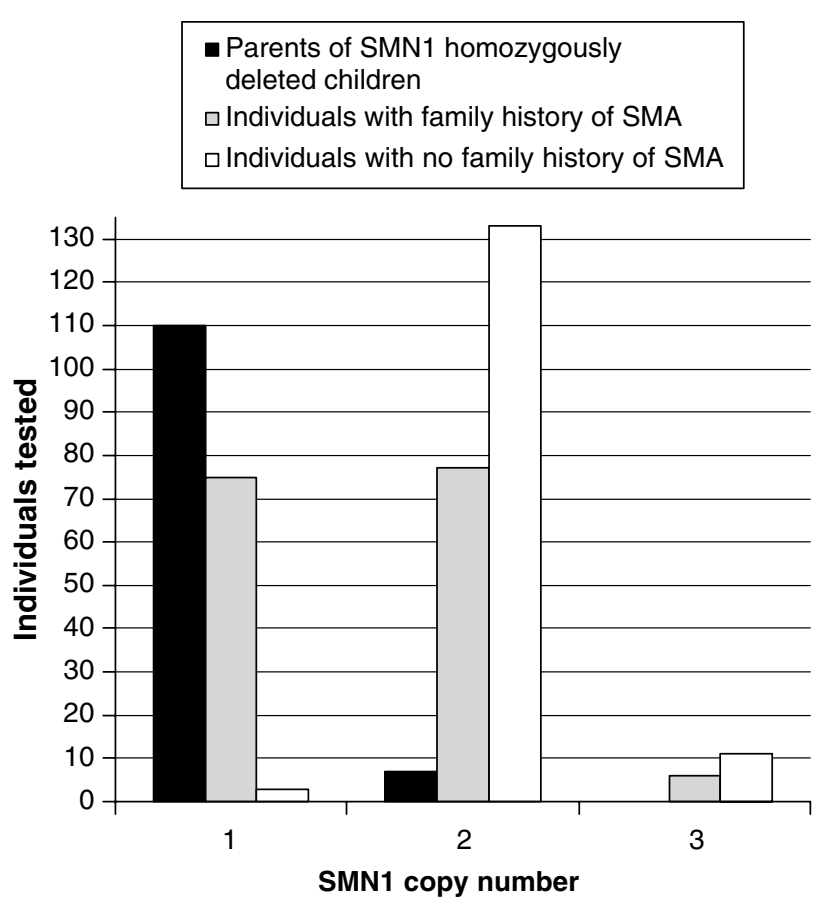

Figure 3 SMN1 gene dosage analysis in the Australian and New Zealand population.

The mother of the affected child in family 3 was found to have two copies of SMN1 (Figure 4). The father and the maternal grandmother of the affected child had only one copy of the SMN1 gene. Phasing of the chromosomes with linkage markers flanking the SMA locus indicated that the mother of the affected child had inherited the SMN1 deleted allele from her mother. Therefore, the other allele from her father carries two copies of the SMN1 gene. In family 4 (Figure 4 ), the mother of the affected children had one copy of the SMN1 gene. Linkage analysis in the affected siblings indicated that the deletion allele was inherited from both parents. Because the deletion was transmitted more than once, the father of the affected child most likely has two copies of SMN1 on one allele.

\section{Discussion}

SMN dosage analysis is used to determine the number of SMN1 gene copies. Because there is great variation in the SMN1 gene copy number between individuals (also highlighted by our data), dosage analysis requires a method that can clearly distinguish between one, two and even three or more copies of SMN1. We have presented a method using quantitative real time PCR technology, which clearly determines the SMN1 copy number in non-overlapping ranges, as shown in Figure 2. A cohort of 422 individuals 

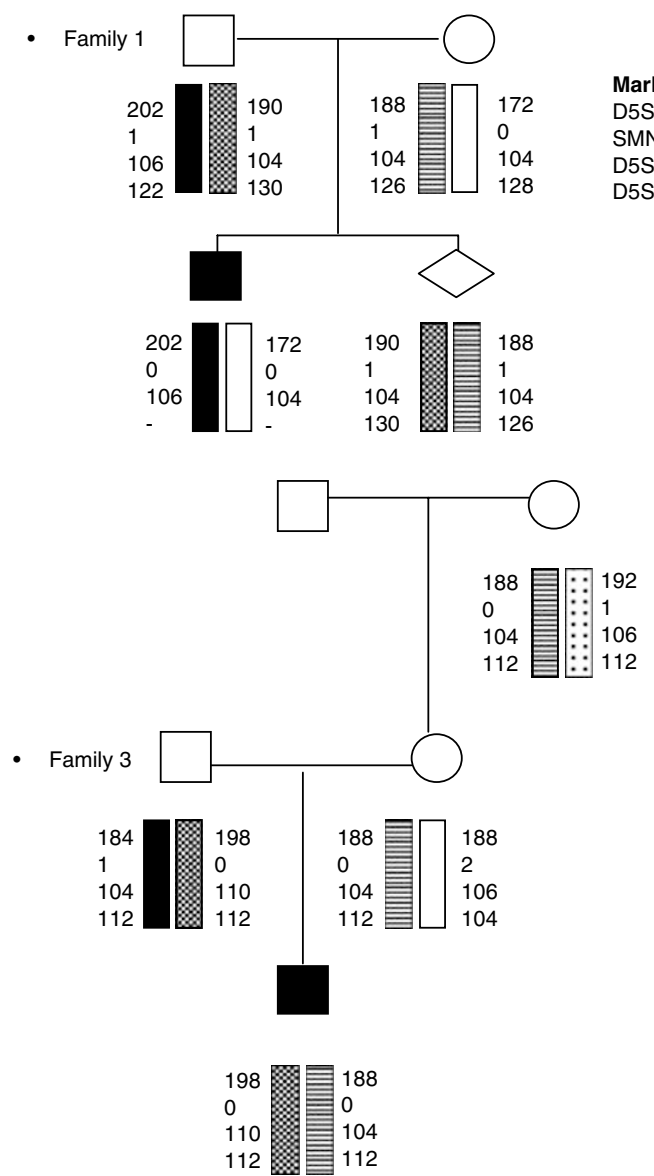

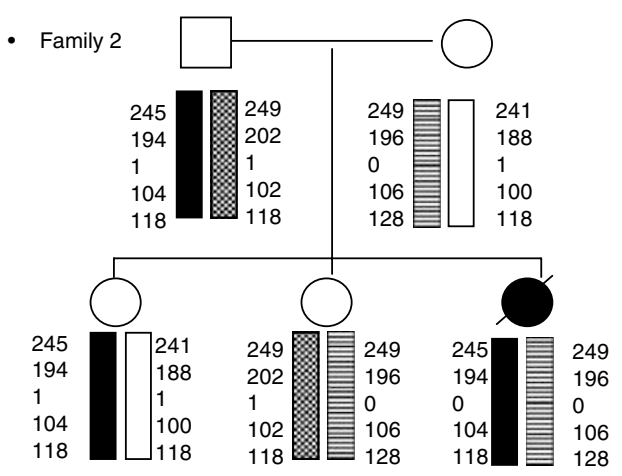

Markers D5S629 D5S1414 SMN1 D5S1408 D5S610 Markers EF1/2a SMN1 D5S1408 JK53

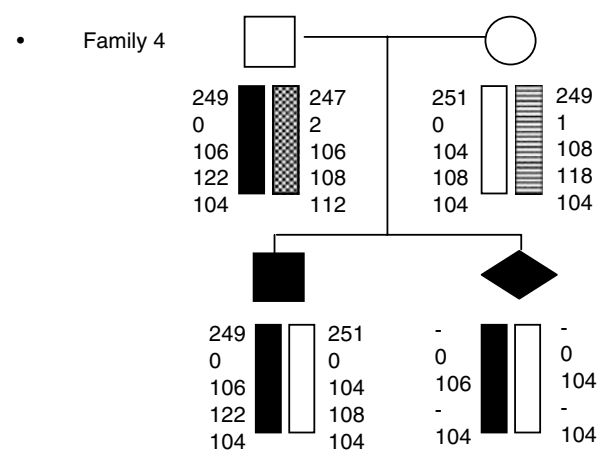

Markers D5S629 SMN1 D5S1408 D5S610 JK53

Figure 4 Four families that underwent SMN1 dosage analysis in combination with SMA linkage analysis. Families 1 and 2 are examples of paternal derived de novo deletions. Families 3 and 4 are examples of two SMN1 copies on one chromosome.

was tested and these data are presented in Figure 3. In the parents, $94 \%$ had only one copy of SMN1 and 6\% had two copies of SMN1. In individuals with a family history of SMA, $47 \%$ had one copy of SMN1, 49\% had two copies of SMN1 and $4 \%$ had three copies of SMN1. In individuals with no family history of SMA, $2 \%$ had one copy of SMN1, $90 \%$ had two copies of SMN1 and $8 \%$ had three copies of SMN1.

\section{SMA carrier frequency}

The SMA carrier frequency in the Australian and New Zealand population appears to be one in 49 , with the identification of three carriers in 147 individuals without a family history of SMA. However, a larger sample size is required to increase the accuracy of this figure. The carrier frequency quoted by other groups varies from $1 / 25$ to $1 / 63$, depending upon the population that is being studied. France is quoted at $1 / 34-1 / 56,{ }^{13,14}$ Germany $1 / 25-1 / 35,{ }^{6,15}$ USA $1 / 40-1 / 60,{ }^{16-18}$ Canada/USA $1 / 54^{19}$ and China $1 /$ $63 .^{20}$ To obtain a more accurate SMA carrier frequency for use in risk calculations, we combined our population data
Table 1 SMN1 copy number among individuals from the general population

\begin{tabular}{lrrcrr}
\hline & \multicolumn{5}{c}{ SMN1 copy number } \\
Authors & 1 & 2 & 3 & 4 & Total \\
\hline Ogino et $a l^{18}$ & 30 & 1080 & 86 & 7 & 1203 \\
Our study & 3 & 133 & 11 & 0 & 147 \\
Total & 33 & 1213 & 97 & 7 & 1350 \\
\hline
\end{tabular}

with the published data, ${ }^{18}$ giving a carrier frequency of 1/41 (Table 1). This combined SMA carrier frequency includes individuals from Germany, France, USA, New Zealand and Australia.

\section{Genotypes in SMA carriers}

In the parent population, carrier status was confirmed in 110 parents (94\%) and seven parents had two copies of SMN1 (6\%). Two of the parents with two copies of the SMN1 gene were fathers with one copy of SMN1 on each 
allele with children who had de novo deletions; both these individuals are therefore not carriers of SMA. Linkage analysis also indicated that the de novo loss of the SMN1 was not due to a recombination event, but most likely due to a gene conversion event of SMN1 into SMN2 in both cases (Figure 4). This gene conversion could be confirmed if SMN2 dosage analysis was performed.

Further, two parents with two copies of the SMN1 gene were shown to each carry two-copies of SMN1 on one allele and no SMN1 copies on the other allele (Figure 4). This is referred to as the ' $2+0$ ' genotype. ${ }^{16}$ These individuals are still carriers of SMA. In family 4 , the deletion mutation was transmitted more than once from the father. The most likely explanation is that he has the ' $2+0$ ' genotype and is a carrier of SMA. Gonadal mosaicism for a SMN1 deletion mutation may also be a possible explanation. ${ }^{21,22}$ However, no cases have been documented, that clearly show this, so it remains a very rare possibility.

The remaining three parents with two copies of SMN1 could not be classified as ' $2+0$ ' SMA carriers or noncarriers with de novo deletions in their children, due to the lack of availability of extended family members for linkage analysis. Monosomal hybrid generation, which would separate the two chromosome $5 \mathrm{~s}$, could enable distinction between ' $2+0$ ' and ' $1+1$ ' genotypes. ${ }^{23}$ However, this type of analysis is not practical in a diagnostic laboratory and linkage analysis still remains the most achievable option.

\section{Observed ' $2+0$ ' genotype frequency}

The ' $2+0$ ' genotype is a fundamental factor that could lead to a false-negative result when testing for SMA carriers. It is therefore essential to determine the frequency of this genotype to include in the risk calculation for SMA carrier testing. The observed ' $2+0$ ' genotype was determined in the parents of affected individuals by performing dosage and linkage analysis on the parents found to have two SMN1 copies. Among the 117 parents, seven had two copies and 110 had one copy of SMN1. Two of these parents were identified as having two SMN1 copies on one allele, that is, ' $2+0$ ' genotype and another two of the parents with two copies of SMN1 were normal that is, one SMN1 copy per allele, ' $1+1$ ' genotype. The remaining three parents' genotypes could not be classified as either ' $2+0$ ' carrier or normal, therefore these individuals were not included in the calculation. Within this group of individuals there were 116 normal alleles that is, at least one copy of SMN1 on chromosome 5 (110 normal alleles in the 110 carrier parents, two normal alleles in the two ' $2+0$ ' parents, plus four normal alleles in the two non-carrier parents), two were 2 SMN1 copy alleles (1.7\%). This frequency could even be as high as $4.2 \%$ if the three unclassified parents' genotypes were identified as ' $2+0$ ' genotype. The ' $2+0$ ' frequency in the Australian and New Zealand population is very similar to other populations when compared with previous quoted frequencies of approximately $2.4-3.3 \% .^{6,24}$

\section{Estimated ' $2+0$ ' frequency}

It is possible to obtain an estimate of the two SMN1 copy allele frequency by studying the general population. This population of individuals is considered to give a more accurate estimate of frequency without any preference towards any specific genotype. Individuals with a family history were not included in the calculation as these individuals could result in a bias in the calculated frequencies. Among the 147 unaffected individuals without a family history of SMA, three had one copy of SMN1, 133 had two copies and 11 individuals had three copies. Therefore of the 291 normal alleles ( 3 normal alleles in the individuals with one SMN1 copy, plus 266 normal alleles in the individuals with two copies, plus 22 normal alleles in the three copy individuals (assuming ' $2+1$ ' genotype)), 11 were two copy alleles (3.8\%). There is a small difference in the observed ' $2+0$ ' frequency in the parent population $(1.7 \%)$ compared with the estimated frequency in the general population (3.8\%). This is most likely due to ascertainment bias as carriers are usually identified only when affected children are born and also three parents with unclassified genotypes could not be included in the calculation. However, to enable accurate risk assessments when offering genetic counselling to individuals, it is necessary to calculate the frequencies of the different copy number SMN1 alleles.

\section{De novo rearrangements frequency}

De novo rearrangements would be a fundamental factor that could lead to a false-positive result when testing for SMA carriers. It is therefore essential to determine the frequency of this event to include it into the risk calculation for SMA carrier testing. In our patient cohort, de novo mutations were detected in two out of 117 parents (1.7\%). This frequency could be as high as $4.3 \%$ if the three parents from the unresolved families were found to have one SMN1 gene on each allele (not SMA carriers). De novo rearrangements are estimated to be found in $\sim 2 \%$ of patients with SMA. ${ }^{7,17,25,26}$ The paternal and maternal de novo mutation rates are estimated to be $2.11 \times 10^{-4}$ and $4.15 \times 10^{-5}$ respectively. ${ }^{18}$ The majority of the reported de novo deletion mutations are paternal in origin, the consequence of a crossover that results in the loss of the SMN1 gene. There have been two reports of the loss of the SMN1 gene due to a de novo gene conversion event, converting a SMN1 gene into SMN2.7,13 Of the reported cases one was of maternal and the other paternal. Both the cases that we identified were paternal in origin. 
Table 2 SMN1 allele and genotype frequencies

\begin{tabular}{lcccc}
\hline Allele/status & Genotype & Gene dosage Designation & $\begin{array}{c}\text { General } \\
\text { population }\end{array}$ \\
\hline 0 copy & & & $a$ & $1.36 \times 10^{-2}$ \\
1 copy & & & $b$ & $9.47 \times 10^{-1}$ \\
2 copy & & & $c$ & $3.99 \times 10^{-2}$ \\
$1^{\mathrm{D}}$ copy & & & $d$ & $2.49 \times 10^{-4}$ \\
Non-carrier & $2+2$ & 4 & $c^{2}$ & $1.59 \times 10^{-3}$ \\
& $2+1$ & 3 & $2 b c$ & $7.56 \times 10^{-2}$ \\
& $1+1$ & 2 & $b^{2}$ & $8.97 \times 10^{-1}$ \\
Carrier & $2+1^{\mathrm{D}}$ & 3 & $2 c d$ & $1.99 \times 10^{-5}$ \\
& $2+0$ & 2 & $2 a c$ & $1.09 \times 10^{-3}$ \\
& $1+1^{\mathrm{D}}$ & 2 & $2 b d$ & $4.72 \times 10^{-4}$ \\
& $1+0$ & 1 & $2 a b$ & $2.58 \times 10^{-2}$ \\
Affected & $1^{\mathrm{D}}+1^{\mathrm{D}}$ & 2 & $d^{2}$ & $6.20 \times 10^{-8}$ \\
& $1^{\mathrm{D}}+0$ & 1 & $2 a d$ & $6.77 \times 10^{-6}$ \\
& $0+0$ & 0 & $a^{2}$ & $1.85 \times 10^{-4}$ \\
\hline
\end{tabular}

\section{What should be considered in genetic risk} assessments?

Genetic risk assessments (GRA) are estimates based on published data from limited patient numbers from specific populations. Carrier frequency, the ' $2+0$ ' genotype, de novo mutation rates and intragenic SMN mutations, are all factors that need to be considered when determining a SMA carrier risk. Individuals with the ' $2+0$ ' genotype are still carriers of SMA and therefore the recurrence risk is high. When a de novo mutation has occurred, one of the parents is not a carrier of SMA and therefore the recurrence risk is low. It is important for parents who have been shown to carry two SMN1 genes, contrary to the expected one SMN1 gene copy, to undergo extended analysis to distinguish between these two events, as it results in a different impact on the potential risk in future pregnancies and family members.

A comprehensive testing procedure for SMA carrier testing requires a multi-modal approach that involves SMN gene-dosage, linkage analysis as well as GRA. ${ }^{22}$ All of these things are integral components that impact on genetic counselling both pre- and post testing.

\section{Updated SMN1 copy number allele frequencies}

The frequency of the different types of SMN1 copy-number alleles is used in GRA. The SMN1 allele frequencies are calculated using the Hardy-Weinberg equilibrium. We have combined our data with individuals in the general population of other published data originating from Germany, France and USA (Table 1$)^{18}$ and data provided on patients with SMA with identifiable mutations in both alleles of SMN1. ${ }^{7}$ The new SMN1 allele frequencies are as follows: $\mathbf{a}$ (the $0-$ copy allele frequency) $=1.36 \times 10^{-2} ; \mathbf{b}$ (the one-copy allele frequency) $=9.47 \times 10^{-1}$; $\mathbf{c}$ (the twocopy allele frequency) $=3.99 \times 10^{-2}$; and $\mathbf{d}$ (the one copy disease allele frequency) $=2.49 \times 10^{-4}$. Refer to Table 2 for SMN1 allele and genotype frequencies.

\section{GRA for population screening}

SMA carrier testing raises many questions when determining if an individual with two or more SMN1 copies is still a SMA carrier. When two copies of the SMN1 gene are identified, this usually indicates that the individual is not a carrier of SMA. However, for a small proportion of individuals $\left((2 a c+2 b d) /\left(b^{2}+2 a c+2 b d\right)=1 / 575\right)$, this result may indicate a ' $2+0^{\prime}$ or ' $1+1^{\mathrm{D}}$ 'genotype and therefore the individual will be a carrier of SMA. When three copies of the SMN1 gene are identified, this is most likely to be a ' $2+1$ ' genotype, with two copies on one allele and one copy on the other, indicating that the individual is not a carrier of SMA. Currently, there is no report of an individual who is a carrier of SMA with a ' $3+0$ ' genotype, but the presence of this genotype has been suggested. ${ }^{18} \mathrm{An}$ extensive array of examples for SMA risk calculations for a range of situations have been described previously in the review by Ogino and Wilson. ${ }^{22}$ Four scenarios are detailed below specifically, addressing the situation of testing individuals from the general population and cascade testing of other family members, using the new SMN1 allele and carrier frequencies.

\section{Scenario 1}

What is the probability of being a SMA carrier if an unaffected individual without a family history of SMA is found to have two copies of SMN1?

The conditional probability of a carrier having two copies of SMN1 is $(2 a c+2 b d) /(2 a b+2 a c+2 b d+2 c d)=$ 0.05694 . The fraction of non-carriers with two copies of SMN1 is $b^{2} / b^{2}+2 b c+c^{2}=0.9206$. By using Bayesian analysis and a $1 / 41$ prior carrier probability $((0.05694 \times 1 /$ $41) /(0.05694 \times 1 / 41+0.9206 \times 40 / 41))$ the posterior carrier probability is $1 / 648$.

\section{Scenario 2}

What is the risk of an individual with two SMN1 copies of having a child with SMA if their partner is a known SMA carrier (one SMN1 copy)?

Using the Bayesian analysis as described in Scenario 1, they have a posterior carrier probability of $1 / 648$. Therefore, the risk of SMA recurrence for this couple, including a de novo mutation rate of $2.11 \times 10^{-4}$ is $((1 / 648 \times 1 / 4)+$ $\left.\left(2.11 \times 10^{-4} \times 1 / 2\right)\right)$, which is approximately $1 / 2035$.

\section{Scenario 3}

What is the risk of a couple without a family history of SMA of having a child with SMA if both individuals have two SMN1 copies?

Using the Bayesian analysis as described in Scenario 1, both individuals have a posterior carrier probability of $1 /$ 648. Therefore, the risk of SMA occurrence for this couple, including a de novo mutation rate of $2.11 \times 10^{-4}$ is $\left((1 / 648 \times 1 / 648 \times 1 / 4)+\left(2.11 \times 10^{-4} \times 1 / 648 \times 1 / 4\right)\right)$, which is approximately $1 / 1500000$. 


\section{Scenario 4}

What is the probability of being a SMA carrier if a child of a SMA carrier with one copy of SMN1 has two copies of SMN1?

Two possibilities must be considered. Possibility 1: The child is not a carrier of SMA because one SMN1 copy from each parent was inherited that is, ' $1+1$ ' genotype; in this situation there is a very small residual risk of inheriting a SMN1 point mutation from the non-carrier parent. Possibility 2: The child is a SMA carrier because the deleted allele was inherited from the carrier parent and a two-copy SMN1 allele was inherited from the non-carrier parent, that is, the ' $2+0$ ' genotype.

Dosage analysis performed in the non-carrier parent could identify 1, 2 or 3 copies of the SMN1. If only one copy is found in the non-carrier parent, the child would be a ' $1+1$ ' genotype and therefore a non-carrier. This confirms possibility 1 . If two copies are detected in the non-carrier parent, then this parent could have one of three possible genotypes: a ' $1+1$ ' genotype (non-carrier) or a ' $2+0$ ' genotype (carrier) or ' $1+1^{\mathrm{D}}$ ' genotype (carrier). The carrier risk of the child is then $1 / 677(2 a c+b d /$ $\left.\left(b^{2}+2 a c+2 b d\right)\right)$. Further linkage and dosage analysis with extended family members are required to resolve the child's carrier status. If three copies are detected in the non-carrier parent, the parent will be a ' $2+1$ ' genotype because the child has not inherited three copies. The ' $2+1$ ' genotype means that the child could have inherited the two copy SMN1 allele from the non-carrier parent and the deleted allele from the carrier parent, and therefore be a ' $2+0$ ' carrier; or be a non-carrier inheriting the one copy from each parent. Further linkage analysis with extended family members is required to resolve the child's carrier status. If the deleted allele were identified in the child, then the ' $2+0$ ' genotype is confirmed. If the nondeleted allele is inherited, then the ' $1+1$ ' genotype is confirmed.

\section{Future testing considerations}

The American Society for Reproductive Medicine has established guidelines for genetic testing of potential gamete donors. Currently, donors are screened for the absence of serious autosomal-recessive hereditary disorders, such as cystic fibrosis, thalassemia and Tay-Sachs disease, depending on the ethnic background of the donor. SMA fits into the category of a serious autosomal-recessive hereditary disorder across all ethnic groups with a high carrier frequency ranging from $1 / 25$ to $1 / 63$. This carrier frequency is in the order of that for cystic fibrosis, which is currently being offered in population screening. Carrier screening for SMA should therefore also be considered in population genetic screening programs for severe genetic disorders.

\section{Conclusion}

Carrier testing for SMA linked to the SMN1 gene can be provided to individuals with or without a positive family history. SMN dosage and linkage analysis combined with GRA offered in conjunction with genetic counselling, can provide the information that will assist individuals to make important choices in their family planning.

\section{Acknowledgements}

We thank Associate Professor Les Sheffield for advice and useful comments on risk calculations.

\section{References}

1 Pearn J: Classification of spinal muscular atrophies. Lancet 1980; 1: $919-922$.

2 Lefebvre S, Burglen L, Reboullet $\mathrm{S}$ et al: Identification and characterization of a spinal muscular atrophy-determining gene [see comments]. Cell 1995; 80: 155-165.

3 Burglen L, Lefebvre S, Clermont O et al: Structure and organization of the human survival motor neurone (SMN) gene. Genomics 1996; 32: 479-482.

4 Kashima T, Manley JL: A negative element in SMN2 exon 7 inhibits splicing in spinal muscular atrophy. Nat Genet 2003; 34: 460-463.

5 Cartegni L, Krainer AR: Disruption of an SF2/ASF-dependent exonic splicing enhancer in SMN2 causes spinal muscular atrophy in the absence of SMN1. Nat Genet 2002; 30: 377-384.

6 Feldkotter M, Schwarzer V, Wirth R, Wienker TF, Wirth B: Quantitative analyses of SMN1 and SMN2 based on real-time lightCycler PCR: fast and highly reliable carrier testing and prediction of severity of spinal muscular atrophy. Am J Hum Genet 2002; 70: 358-368

7 Wirth B, Herz M, Wetter A et al: Quantitative analysis of survival motor neuron copies: identification of subtle SMN1 mutations in patients with spinal muscular atrophy, genotype-phenotype correlation, and implications for genetic counseling. Am J Hum Genet 1999; 64: 1340-1356.

8 Burghes AHM: When is a deletion not a deletion? When it is converted. Am J Hum Genet 1997; 61: 9-15.

9 Wirth B: An update of the mutation spectrum of the survival motor neuron gene (SMN1) in autosomal-recessive spinal muscular atrophy (SMA). Hum Mutat 2000; 15: 228-237.

10 Ogino S, Wilson RB: Spinal muscular atrophy: molecular genetics and diagnostics. Expert Rev Mol Diagn 2004; 4: 15-29.

11 Prior TW, Swoboda KJ, Scott HD, Hejmanowski AQ: Homozygous SMN1 deletions in unaffected family members and modification of the phenotype by SMN2. Am J Med Genet A 2004; 130: 307310.

12 Scheffer H, Cobben JM, Matthijs G, Wirth B: Best practice guidelines for molecular analysis in spinal muscular atrophy. Eur J Hum Genet 2001; 9: 484-491.

13 Cusin V, Clermont O, Gerard B, Chantereau D, Elion J: Prevalence of SMN1 deletion and duplication in carrier and normal populations: implication for genetic counselling. I Med Genet 2003; 40: e39.

14 Corcia P, Mayeux-Portas V, Khoris J et al: Abnormal SMN1 gene copy number is a susceptibility factor for amyotrophic lateral sclerosis. Ann Neurol 2002; 51: 243-246.

15 Anhuf D, Eggermann T, Rudnik-Schoneborn S, Zerres K: Determination of SMN1 and SMN2 copy number using TaqMan technology. Hum Mutat 2003; 22: 74-78.

16 Chen KL, Wang YL, Rennert $\mathrm{H}$ et al: Duplications and de novo deletions of the SMNt gene demonstrated by fluorescence-based 
carrier testing for spinal muscular atrophy. Am J Med Genet 1999; 85: $463-469$.

17 Ogino S, Leonard DG, Rennert H, Ewens WJ, Wilson RB: Genetic risk assessment in carrier testing for spinal muscular atrophy. Am J Med Genet 2002; 110: 301-307.

18 Ogino S, Wilson RB, Gold B: New insights on the evolution of the SMN1 and SMN2 region: simulation and meta-analysis for allele and haplotype frequency calculations. Eur J Hum Genet 2004; 12: $1015-1023$.

19 McAndrew PE, Parsons DW, Simard LR et al: Identification of proximal spinal muscular atrophy carriers and patients by analysis of SMNT and SMNC gene copy number. Am J Hum Genet 1997; 60: 1411-1422.

20 Chan V, Yip B, Yam I et al: Carrier incidence for spinal muscular atrophy in southern Chinese. J Neurol 2004; 251: 1089-1093.

21 Campbell L, Daniels RJ, Dubowitz V, Davies KE: Maternal mosaicism for a second mutational event in a type I spinal muscular atrophy family. Am J Hum Genet 1998; 63: 37-44.
22 Ogino S, Wilson RB: Genetic testing and risk assessment for spinal muscular atrophy (SMA). Hum Genet 2002; 111: 477-500.

23 Mailman MD, Hemingway T, Darsey RL et al: Hybrids monosomal for human chromosome 5 reveal the presence of a spinal muscular atrophy (SMA) carrier with two SMN1 copies on one chromosome. Hum Genet 2001; 108: 109-115.

24 Ogino S, Wilson RB: SMN dosage analysis and risk assessment for spinal muscular atrophy. Am J Hum Genet 2002; 70: 1596-1598; author reply 1598-1599.

25 Wirth B, Schmidt T, Hahnen E et al: De novo rearrangements found in $2 \%$ of index patients with spinal muscular atrophy: mutational mechanisms, parental origin, mutation rate, and implications for genetic counseling. Am J Hum Genet 1997; 61: $1102-1111$

26 Melki J, Lefebvre S, Burglen L et al: De novo and inherited deletions of the $5 \mathrm{q} 13$ region in spinal muscular atrophies. Science 1994; 264: 1474-1477. 